\title{
Influencia del Liderazgo docente en la motivación de universitarios
}

\section{Influence of the teaching Leadership in the motivation of university students}

\section{RESUMEN.}

Juan Velázquez Hernández. Universidad Juárez Autónoma de Tabasco. hernandez.2707juan@hotmail.com

Gladys Hernández Romero. Universidad Juárez Autónoma de Tabasco. gladiolita6@hotmail.com

Este trabajo presenta los resultados de un estudio sobre la influencia del liderazgo docente en la motivación de los jóvenes universitarios, en la División Académica de Ciencias Económico Administrativas, perteneciente a la Universidad Juárez Autónoma de Tabasco, en México. Las evidencias aquí mostradas, son el resultado de 120 estudiantes de la licenciatura en Administración, el instrumento utilizado para la medición fue de un cuestionario en la escala de Likert, aplicados durante el periodo de Agosto 2017-Enero 2018. Los resultados apuntan que existen mayores niveles de motivación cuando el liderazgo es de tendencia democrática, no obstante, es necesario que todos los participantes de la pirámide educativa se comprometan y busquen las herramientas necesarias para formar profesionistas íntegros que la sociedad demanda.

\section{PALABRAS CLAVE.}

Educación Superior, motivación, labor educativa, Liderazgo, universitarios.

\section{ABSTRACT.}

This paper presents the results of a study on the influence of teacher leadership on the motivation of university students, of Division Academica de Ciencias Economico Administrativas from Universidad Juarez Autonoma de Tabasco, in Mexico. The evidences how here is the result of 120 undergraduate students in Administration, the instrument used for the measurement was a questionnaire on the Likert scale, applied during the period of August 2017 to January 2018. The information obtained that there are higher levels of motivation when the leader ship is democratic, however, it is necessary that all participants in the educational pyramid are committed and seek the necessary tools to train integral professionals that society demands.

\section{KEY WORDS.}

Higher education, motivation, educational work, Leadership, university students 


\section{Introducción.}

El conocimiento es la herramienta principal para el progreso de las sociedades, hoy en día actualizar los procesos de enseñanza-aprendizaje, resulta cada vez más importante para desarrollar las competencias.

El papel que desempeñan los profesores es tan relevante en la pirámide educativa, al ser los impulsores de la educación, tienen diferentes responsabilidades, desde proporcionar información oportuna, clara y concisa, hasta impulsar a los jóvenes a ser mejores ciudadanos, comprometidos con su entorno.

La práctica profesional requiere de futuros egresados que resuelvan problemas de distinta índole, las instituciones de educación superior deben formar profesionales que se conviertan en líderes sociales, emprendedores y capaces de diseñar planes de acción en busca de soluciones.

Prieto (2008), menciona que el "Docente dentro de su experiencia educativa, toma en cuenta las diferentes costumbres, creencias, certezas y escalas de valor que, de cierta forma, implementara en su plan de trabajo con sus aprendices" (p. 328).

Partiendo de lo anterior, el profesor debe impactar positivamente en cada dimensión de la formación integral de los futuros profesionistas, para esto, se requiere de la presencia del liderazgo docente, ya que, tienen en sus manos la capacidad de dirigir, controlar, guiar e influir en los alumnos por medio de sus enseñanzas.

El maestro puede establecer el camino de la excelencia o del descenso académico a través del estilo de liderazgo que adopte, por otra parte, la motivación es un factor esencial para la persecución de las metas, lo cual permite que los aprendices sean más receptivos y eficaces.

La motivación académica establece uno de los componentes psicoeducativos que más influencia tiene en el aprendizaje. No consiste solamente en poner técnicas o métodos, sino va más allá de lo escolar, la motivación tiene una compleja interrelación con los factores cognitivos, afectivos, sociales y por supuesto académicos; y de cierta manera se relaciona con las acciones del profesor-alumno (Expósito \& Manzano, 2010: 334).

Para contribuir a que los estudiantes se sientan motivados para conocer, conlleva la existencia de ellos en total claridad y coherencia, en cuanto al objetivo de los procesos de aprendizaje, que se sientan inspirados y capaces de resolver los retos que se les presente (Ospina, 2006: 160).

No obstante, los distintos tipos de liderazgo que ejerzan los profesores siempre tendrán repercusiones en los estudiantes, ya sea para bien o para mal. El guía no debe limitarse a ser un facilitador de conocimientos, sino inducir a cada uno de sus estudiantes en busca de sus objetivos personales, promoverles la creatividad y a que tengan un pensamiento críticoreflexivo.

Esta investigación ha sido diseñada para saber cómo influye el liderazgo docente en la motivación de los alumnos de la DACEA-UJAT, con este estudio se pretende demostrar que el maestro más que un generador de conocimientos es un modelo a seguir en la vida del estudiante.

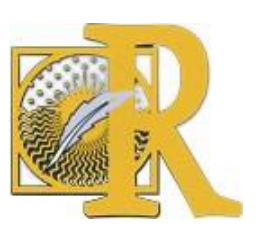




\section{Desarrollo.}

Una de las herramientas imprescindibles para guiar a las personas es el liderazgo, la influencia que puede ejercer un líder sobre un grupo de personas abarca desde la manera de pensar hasta la de actuar.

Las organizaciones son espacios productivos, que buscan la persecución de las metas establecidas, para lo cual se requieren de personas aptas para cumplir con las tareas asignadas, un factor decisivo en la productividad es la motivación.

En el ámbito de la educación, los docentes figuran como líderes, al tener el mando y control sobre su grupo, donde el objetivo principal es lograr que sus estudiantes se nutran de conocimientos y sean competitivos, el ser docente requiere de mucha responsabilidad, que marque el rumbo de la sociedad, al formar líderes que la nación necesita.

Los maestros al controlar los procesos de enseñanza-aprendizaje, pueden impactar en las motivaciones, emociones y actitudes de sus aprendices; siendo la motivación el principal factor para lograr los objetivos deseados.

Una de las señales educativas más importantes para favorecer el aprendizaje es que los alumnos estén interesados y motivados por conocer lo que soliciten. Los estudiantes valoran lo que aprenden cuando comprenden y perciben su significado, cuando aprecian su relevancia y pertinencia, así como la utilidad que tiene para la sociedad y su vida profesional (Tirado, Santos \& Tejero-Díez, 2013: 80).

Al respecto González \& González (2012: 36) manifiestan que los profesores deben motivar a sus estudiantes en la persecución de los objetivos trazados, por lo que su preparación debe centrarse en dar respuestas al contexto educativo por medio de la motivación y el estímulo constante, brindando confianza y seguridad en los estudiantes.

La efectividad del profesor depende del entusiasmo, de la dedicación y de la orientación adecuada en las tareas que desarrolla en el aula. Además, es indispensable que proporcione actividades recreativas, junto a una evaluación individual adecuada, por lo que, el maestro, debe prepararse para enriquecer los conocimientos de sus estudiantes (Cermeño, 2016: 15).

Los profesores no solo tienen la capacidad de satisfacer las necesidades básicas de sus aprendices, sino también originan cuatro condiciones indispensables para el ser humano: el comportamiento, el respeto, la empatía e impulsar para crecer y mejorar (Panta, 2010:17).

El estilo de liderazgo del docente puede impactar de manera positiva o negativa en los diferentes ámbitos de la vida del estudiante, un equipo de guías educativos y de alumnos inspirados a ser mejores personas, motivados a aprender, podrán conseguir las metas propuestas, sin importar las situaciones vulnerables en las que se encuentren.

El buen líder busca desarrollar las capacidades de sus seguidores, de tal manera que puedan obtener aquellos estímulos que son valorados por ellos, y a su vez permite se produzca un proceso motivacional, que trascienda la búsqueda de un fin individual, asegurando que el interés perseguido sea de carácter colectivo y solidario (Silva, Gandoy, Jara \& Pacenza, 2015: 70).

La experiencia que el profesor posee se convierte para el estudiante una herramienta de aprendizaje, debido a que ve en ella un punto de encuentro como profesional que comparte intereses, vivencias, permitiendo establecer motivaciones en él, por conocer lo vivido por su

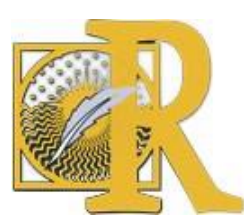


docente en situaciones que esperan sucedan en futuro, en su formación profesional (Ospina, Toro \& Aristizábal, 2008: 112).

En el entorno educativo se requiere de responder a las interrogantes qué, para qué, por qué, al cómo y al hacia dónde. Estas preguntas definen una propuesta innovadora, que es consecuente con las necesidades del contexto. Es necesario preparar a los jóvenes más para la vida, para el mundo, para actuar de manera correcta y transformar a otros (Sierra, 2016: 114).

Uno de los aspectos relevantes de las Instituciones de Educación Superior, es que permiten el ejercicio del liderazgo en las áreas académicas, en la toma de decisiones, en la coordinación de equipos y en los proyectos a la comunidad estudiantil (Rivera \& Cavazos, 2015: 373).

El liderazgo ha evolucionado debido a que ha generado mayor comprensión en los efectos que provoca en otras personas y también las exigencias de las Instituciones educativas son cada vez mayores, desde alcanzar las metas curriculares hasta formar profesionistas altamente competitivos (Amador, 2018: 829).

La motivación es el motor de la conducta humana. El interés por una actividad es "despertado" por una necesidad, la misma que es un mecanismo que incita a la persona a la acción, y que puede ser de origen fisiológico o psicológico. Cada vez que aparece una necesidad, ésta rompe el estado de equilibrio del organismo y produce un estado de tensión, insatisfacción e inconformismo que lleva al individuo a desarrollar un comportamiento o acción (Carrillo, Padilla, Rosero \& Villagómez, 2009: 21).

Los docentes deben ser capaces de impulsar a los estudiantes hacia el aprendizaje, no obstantes, para exigir la motivación escolar, es necesario formar motivacionalmente a los futuros profesores, no basta con el carisma personal, sino contar con conocimientos adecuados sobre los estímulos que sirva como referencia y guíen los esfuerzos de los docentes (Valenzuela, Muñoz, Silvia-Peña, Gómez \& Precht, 2015: 356)

El proceso motivacional es el resultado de una combinación de factores asociados con dos dimensiones personales: internas, ligada a los intereses, deseos y expectativas de las personas; y la externa, referente a los aspectos del entorno que pueden funcionar como estímulos o recompensas (Steinmann, Bosch \& Aiassa, 2013: 586).

La motivación es intrínseca cuando nace de la propia persona y su relación con la actividad y es extrínseca cuando los factores del exterior impulsan al individuo a la realización de una actividad establecida.

En esta investigación se busca analizar los estilos de liderazgo de los docentes, lo cual ofrece una aproximación al conocimiento de su proprio comportamiento y saber cómo influye en la motivación del estudiante, los estilos a presentar son: el democrático, autocrático y liberal.

Un guía democrático escucha a sus aprendices y valora su opinión "El líder democrático da pautas generales a modo de guía, tomando en cuenta las opiniones e ideas de sus seguidores, efectúa indicaciones y sugerencias básicas para lograr las metas grupales" (Soria, 2014). El guía al escuchar diferentes puntos de vista, va fortaleciendo su formación profesional. Un docente democrático, motivará constantemente a su grupo, aceptando ideas, opiniones o sugerencias de los demás la cual influirá en su toma de decisiones.

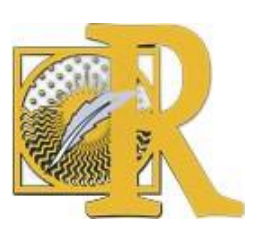


El líder autocrático, ejerce la acción del mando, no trata de interesarse por saber qué es lo que piensa su grupo, solo se enfoca en darles órdenes que deben de seguir sin discusión alguna, los colaboradores deben de seguirlos por obediencia y en forma pacífica (Ponce, 2008: 10). Este tipo de líder dirige de forma rígida, su autoridad puede ocasionar temor entre el grupo, por lo que pide obediencia y cumplimiento de las tareas.

El liderazgo Liberal se caracteriza por entregarles a sus subordinados la autoridad para tomar decisiones. Espera que los integrantes del equipo asuman la responsabilidad por su propia motivación, guía y control, por lo que ellos deben de estar altamente calificados para obtener resultados satisfactorios. (Flores, 2010).

\section{Metodología.}

El universo en este trabajo de investigación es de 174 estudiantes inscritos en el periodo Agosto 2017-Enero 2018, que cuenta por lo menos con el 50\% de avance curricular.

Esta población corresponde a la licenciatura de Administración, perteneciente a la División Académica de Ciencias Económico Administrativas, en la ciudad de Villahermosa, Tabasco, México. En la siguiente tabla se puede observar dicha información.

Tabla 1. Distribución de la población de la Lic. En Administración

\begin{tabular}{|l|l|}
\hline \multicolumn{2}{|c|}{ Lic. en Administración } \\
\hline Avance curricular & Alumnos \\
\hline 50 a $59 \%$ & 27 \\
\hline 60 a $69 \%$ & 29 \\
\hline 70 a $79 \%$ & 35 \\
\hline 80 a $89 \%$ & 38 \\
\hline 90 a $99 \%$ & 45 \\
\hline Total & 174 \\
\hline
\end{tabular}

Fuente: elaboración propia.

Tamaño de la muestra.

En esta investigación se define a la muestra como una representación de la población. La muestra es "en esencia, un subgrupo de la población, digamos que es subconjunto de elementos que pertenecen a ese conjunto definido en sus características al que llamamos población" (Hernández \& et al, 2006: 240), la cual tiene características definidas, este estudio estuvo conformado por 174 estudiantes. 
La fórmula para calcular el tamaño de la muestra, es el siguiente:

Donde

$$
n n=\frac{N N(Z Z)^{2}(50 \%)^{2}}{(N N-1)(e e)^{2}+(z Z)^{2}(50 \%)^{2}}
$$

$\mathrm{n}=$ Tamaño de la muestra

$\mathrm{N}=$ Tamaño de la población

$Z=$ Nivel de confianza

$\mathrm{e}=$ Margen de error

$50 \%=$ Probabilidad de éxito

$n n=\frac{174(1.96)^{2}(.50)^{2}}{(1431-1)(0.05)^{2}+(1.96)^{2}(.50)^{2}}$

$n n=\frac{174(3.8416)(0.25)}{174(0.0025)+3.8416(0.25)}$

$n n=\frac{174(0.9604)}{0.435+0.9604}$

$n n=\frac{167.1096}{1.3954}$

$n n=120$ EEEEEEEEEEEEEnnEEeeEE

Como instrumento se utilizará un cuestionario estructurado, el cual tendrá un formato en escala de Likert, donde tendrá 5 categorías de respuestas (Muy de acuerdo a Muy en desacuerdo).

Las respuestas harán referencia al estilo de liderazgo del docente; las características fueron planteadas en el apartado anterior. De esta manera, se podrá conocer cómo influye de manera positiva, intermedia y negativa los estilos de liderazgo en la motivación de los estudiantes en administración. 


\section{Resultados.}

Es de todos conocidos, que la educación juega un papel imprescindible en el desarrollo de una sociedad, y con ella, la relación del docente-alumno representa una probabilidad de elevar el nivel académico en los centros de enseñanza.

En la era del conocimiento se exigen cambios en los paradigmas que superen los planes curriculares, los docentes deben preparar sus clases con anticipación, utilizando los recursos didácticos y tecnológicos, de tal manera que sus estudiantes analicen los temas y se nutran de información. El profesor como tiene una comprensión más completa y compleja de los temas a tratar, realiza una adaptación para presentar una versión simplificada que pueda entrar en intersubjetividad con el nivel de entendimiento de los estudiantes (Goldrine \& Rojas, 2007). En la siguiente gráfica el $75 \%$ de los estudiantes respondió estar muy de acuerdo y de acuerdo en que el maestro lleva a cabo una introducción de los temas que se abordarán en clases, apuntando hacia las características del líder democrático.

\section{Gráfica 1. El profesor explica de qué tratarán los temas que se abordarán en clases.}

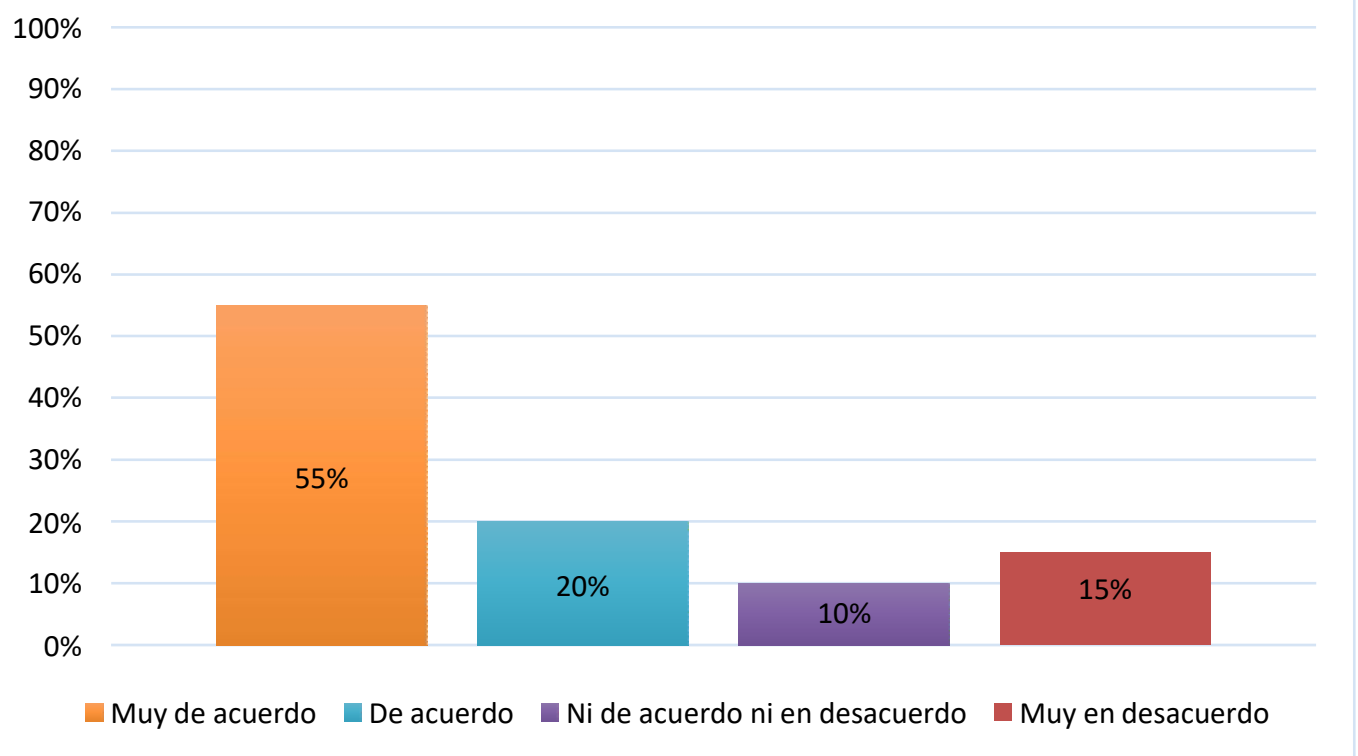

Fuente: elaboración propia.

En la siguiente gráfica, se puede observar que el $65 \%$ de los encuestados respondió estar muy de acuerdo y de acuerdo en que el docente realiza preguntas para despertar la atención de los estudiantes, haciendo referencia al liderazgo democrático. Los buenos profesores tienen como objetivo que sus aprendices se interesen en la clase por medio de la participación, esta técnica estimula la creatividad e impulsa el raciocinio, un buen líder hace preguntas reflexivas y abiertas que se contesten con respuestas justificadas (Gutiérrez, 2008: 1300-1301). El profesor democrático siempre estará dispuesto a escuchar, a ayudar a sus aprendices, a conocer sus opiniones, a intercambiar ideas, independientemente de la situación en la que se encuentren.

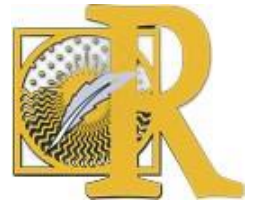


Gráfica 2. El maestro realiza preguntas para despertar el interés en las clases.

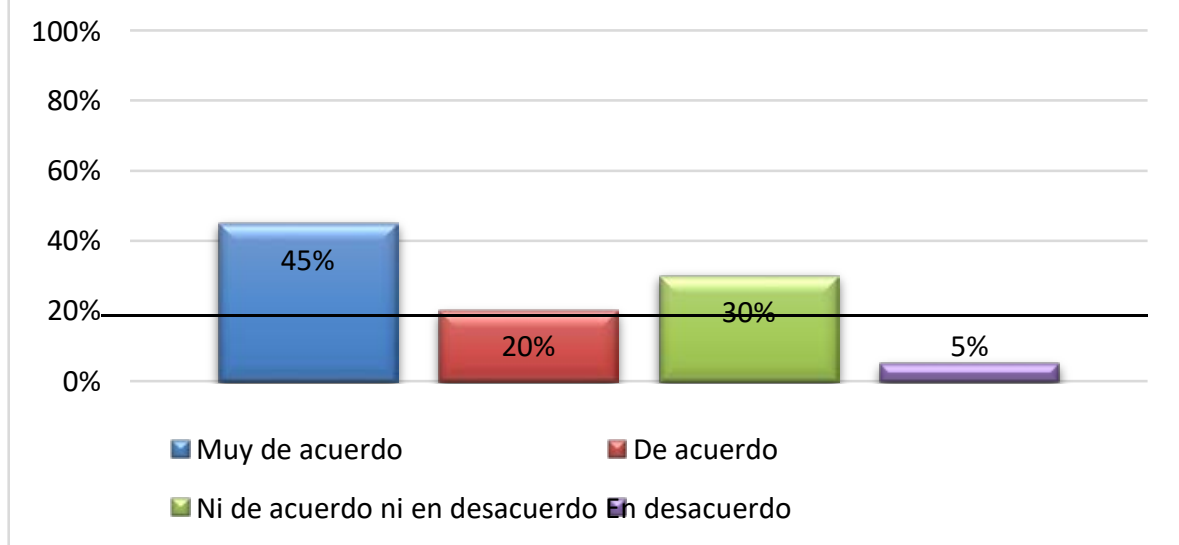

Fuente: elaboración propia.

Cortés señala por ello un buen liderazgo debe mejorar la capacidad del grupo para realizar las aspiraciones y llegar a ser una organización en la que los trabajadores puedan realizar su labor con satisfacción..." (2005: 205). Los docentes democráticos buscan impulsar a sus alumnos, guiándolos, enseñándolos, animándolos, a que confíen y crean en su potencial, además de conducirlos al logro de las metas, de esta manera, el $55 \%$ de los futuros administradores contestó que sus docentes los motivan al logro de sus actividades. Inspirar a los aprendices conlleva a despertar su atención, alentarlos a la persecución de sus objetivos a corto, mediano y largo plazo, además de estimularlos a valorar su constante esfuerzo y explicarles que son capaces de alcanzar el éxito, un estudiante motivado, será capaz de terminar sus actividades, de asimilar los conocimientos y ponerlos en práctica.

Gráfica 3. El docente te motiva al logro de tus actividades.

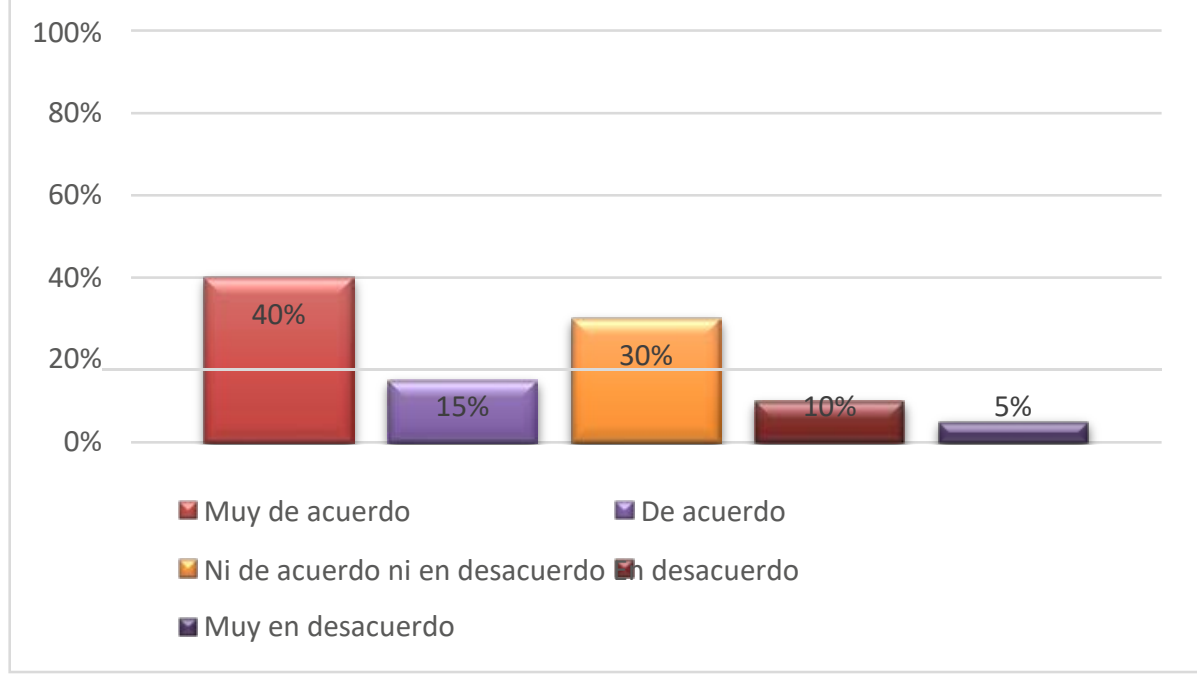

Fuente: elaboración propia.

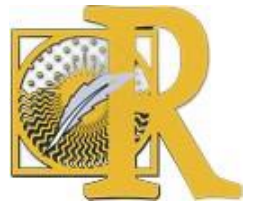


La motivación es considerada como un estado de ánimo, que se puede fortalecer en las organizaciones por medio de los líderes. Un equipo conformado por docentes líderes y alumnos motivados al aprendizaje, podrán alcanzar las metas establecidas, sin importar las situaciones desfavorables (Rojas \& Gaspar, 2006: 120). A la pregunta sobre si el profesor inspira a los futuros administradores a que confíen en ellos y en sus habilidades el $45 \%$ respondió estar muy de acuerdo y de acuerdo, refiriéndose al guía democrático, en cambio, el $40 \%$ optó por la respuesta neutral, señalando de esta manera a los líderes autocráticos y el resto a los guías liberales.

La confianza genera un ambiente armónico, que facilita la comunicación y entendimiento, además de fortalecer el trabajo en equipo e impulsa la toma de decisiones, un líder democrático obtiene el respeto y la confianza de sus aprendices, logra que sigan sus ideales y que cumplan con las actividades asignadas.

Tabla 2. El profesor te inspira a que confíes en ti y en tus habilidades.

\begin{tabular}{|l|l|r|r|}
\hline & Frecuencia & Porcentaje \\
\hline Válido & $\begin{array}{l}\text { Muy de } \\
\text { acuerdo }\end{array}$ & $32 \%$ \\
\hline De acuerdo & 12 & $10 \%$ \\
\hline $\begin{array}{l}\text { Ni de } \\
\text { acuerdo ni } \\
\text { en } \\
\text { desacuerdo }\end{array}$ & 48 & $40 \%$ \\
\hline Desacuerdo & 12 & $10 \%$ \\
\hline $\begin{array}{l}\text { Muy en } \\
\text { desacuerdo }\end{array}$ & 6 & $5 \%$ \\
\hline Total & 120 & 100 \\
\hline
\end{tabular}

Fuente: elaboración propia.

El conocimiento sobre la enseñanza que pueden tener los profesores líderes no se puede explicar por medio de un conjunto de afirmaciones y prescripciones; este tipo de conocimiento está estrechamente relacionado con las experiencias y los contextos en que ocurren (Castellanos \& Yaya, 2013). Un docente que utiliza sus experiencias como ejemplos en clases, es una de las mejores herramientas para acaparar la atención de sus estudiantes, de acercarlos más a la realidad y motivarlos al desarrollo de sus actividades. En la siguiente gráfica se puede observar que el $45 \%$ está muy de acuerdo y de acuerdo en que sus docentes utilizan esta técnica, apuntando hacia el liderazgo democrático, asimismo, un $45 \%$ contestó la opción neutral, aludiendo a los líderes autocráticos. 
Gráfica 4. El docente utiliza anécdotas o vivencias en sus explicaciones.

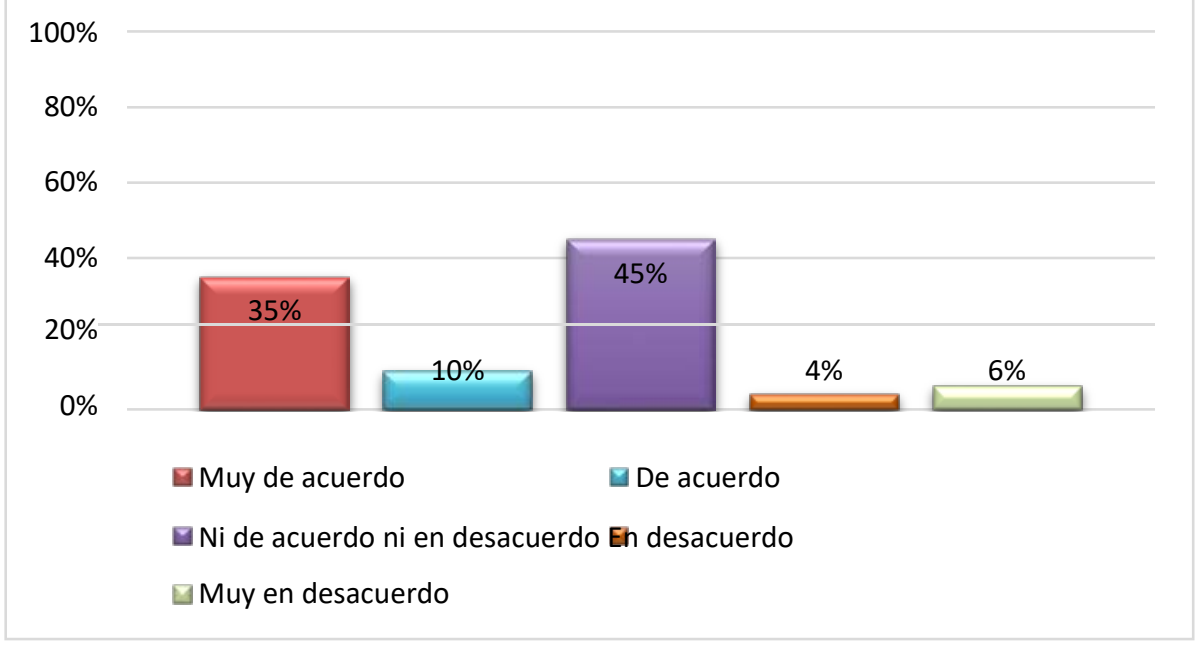

Fuente: elaboración propia.

Al respecto Agustín (2014: 17) considera que el profesor como líder debe enseñar con valores, con responsabilidad y compromiso, al ser un modelo, sus aprendices pueden seguir sus pasos, la formación integral de sus estudiantes se puede obtener a través del desarrollo de prácticas y valores, necesarias para promover una sociedad productiva. Cuando a los futuros administradores se les cuestionó sobre si sus docentes son tolerantes y respetuosos ante respuestas equívocas el $50 \%$ respondió estar de acuerdo y muy de acuerdo, aludiendo a docentes democráticos capaz de respetar las opiniones de los demás. El docente al poner en práctica los derechos universales, crea un ambiente cálido entre sus seguidores: el líder democrático es humanista, accesible y establece confianza y cordialidad con el objetivo de potenciar el desarrollo emocional e intelectual de cada uno de sus alumnos, de este modo, se promueven las cualidades individuales, en busca de una convivencia pacífica.

Tabla 3. El docente es tolerante y respetuoso ante una respuesta errónea.

\begin{tabular}{|c|c|c|c|}
\hline 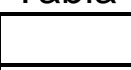 & 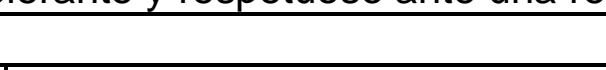 & Frecuencia & Porcentaje \\
\hline \multirow{6}{*}{ Válido } & Muy de acuerdo & 48 & $40 \%$ \\
\hline & De acuerdo & 12 & $10 \%$ \\
\hline & Ni de acuerdo ni en desacuerdo & 36 & $30 \%$ \\
\hline & Desacuerdo & 18 & $15 \%$ \\
\hline & Muy en desacuerdo & 6 & $5 \%$ \\
\hline & Total & 120 & 100 \\
\hline
\end{tabular}

Fuente: elaboración propia. 
Es necesario que los futuros profesionistas tengan una formación sólida con una visión amplia, con sentido humanitario, que actúe con lealtad y responsabilidad en las organizaciones, no es suficiente con tener una preparación técnica; las instituciones como sus integrantes deben ser transparentes, puesto que ambas son percibidas por su entorno (Vílchez, 2012: 245).

Un alumno motivado, será capaz de trabajar de manera independiente, será responsable, creerá en sí mismo y conseguirá mejores resultados en sus trabajos. A la pregunta sobre si el docente los anima a tener éxito en la vida, el $75 \%$ contestó estar muy de acuerdo y de acuerdo, apuntando hacia el liderazgo democrático.

La fortaleza y el éxito de la educación proviene de los docentes, el líder que anima constantemente a sus seguidores y que les ofrece las herramientas didácticas necesarias para impulsar el rendimiento académico, los encamina al logro de sus proyectos, un profesor democrático será capaz de formar a profesionistas autónomos, comprometidos a solucionar con pasión, con ético y con justicia los problemas de la sociedad.

Gráfica 5. El profesor te ánima a tener éxito en tu vida.

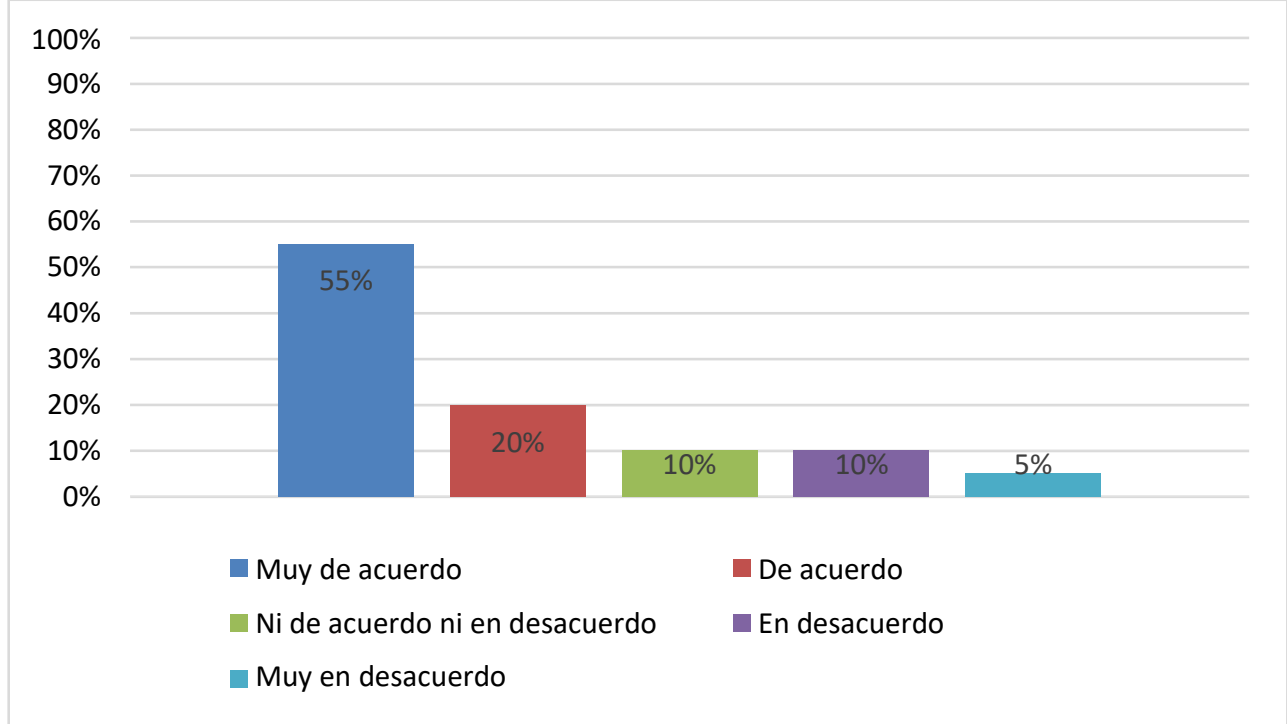

Fuente: elaboración propia.

\section{Conclusiones.}

La educación es imprescindible para el desarrollo económico, político y social de un país; para lograr un aprendizaje significativo es necesario que los centros de enseñanzas cuenten con profesores íntegros, capaces de impulsar la participación analítica, en la búsqueda de soluciones para solventar los problemas que la nación presenta.

Un correcto liderazgo educativo se caracteriza principalmente por una estimulación que propicie a los estudiantes al logro de sus objetivos tanto personales como académicos, permitiendo que canalicen su esfuerzo, capacidad y creatividad.

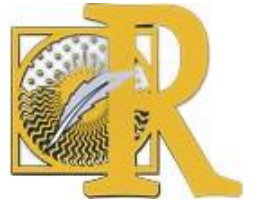


Es necesario destacar que para promover un liderazgo eficiente se requiere que las competencias directivas se ajusten a un plan educativo integral de tal forma que impacten en los principios de sus aprendices.

El buen liderazgo se centra en el sentido humanitario, trascendiendo a lo profesional, por lo tanto, es el guía quien inspira confianza y respeto entre sus seguidores, además de tomarlos en cuenta en la toma de decisiones bajo el concepto de valores.

El profesor es el principal modelo a seguir de sus aprendices, una palabra o un gesto pueden motivar o inhibir en el grupo. El papel del guía es establecer un ambiente positivo y emocional que abra paso a la seguridad, a la motivación y al diálogo, donde todos los estudiantes se sientan seguros de sí mismos para impulsar su rendimiento académico.

El liderazgo democrático suele ser el más efectivo en las organizaciones y por ende conduce al éxito, aportando grandes beneficios, permitiendo que las ideas fluyan y se intercambien de forma libre, abriendo paso al debate y a la discusión, no obstante, el guía es quien tendrá la decisión final en relación a los comentarios de los demás, lo que le permitirá controlar los resultados.

No obstante, un líder autoritario no podrá optimizar el potencial de sus alumnos debido a que muestra un dominio total del grupo propiciando temor entre los participantes, al respecto los guías liberales se deslindan de las responsabilidades, imposibilitando que el equipo potencie sus habilidades, ya que no demuestra interés en el proceso de enseñanzaaprendizaje, solo se enfoca en los resultados, siendo el estilo menos adecuado en la formación del estudiante.

En este estudio se puede constatar, que el estilo de liderazgo del docente que tiene mayor impacto es el democrático, al contribuir a mejorar el estado de ánimo de sus seguidores, de que se sientan dentro del grupo y reconocerles el valor que tienen como personas, por lo tanto, la Universidad Juárez Autónoma de Tabasco cuenta con docentes de este estilo, alineándose a la misión de formar profesionistas capaces de adquirir, generar, difundir y aplicar el conocimiento científico, tecnológico y humanista, con ética y responsabilidad para ser mejores individuos y ciudadanos.

Los futuros egresados de la Licenciatura en Administración serán profesionistas comprometidos con el progreso de la sociedad, con conocimientos y capacidades en las disciplinas de las áreas de administración, contabilidad, finanza, mercadotecnia y economía.

Esta investigación abre paso a futuros estudios desde otras variantes, ya sea de tipo cualitativo o desde la perspectiva docente, para comprender las situaciones particulares de la gestión y liderazgo de las diversas instituciones educativas. 


\section{Referencias bibliográficas.}

- Agustín, G. (2014). Liderazgo docente y disciplina en el aula. Tesis de grado para obtener el título de Licenciado en Pedagogía con Orientación en Administración y Evaluación Educativas, Facultad de Humanidades, Universidad Rafael Landívar, Quetzaltenango, Guatemala. Recuperado de: http://bibliod.url.edu.gt/F/YIV7N511EFP95BF68IJ874LUMNPF26PC2T55174DH7A6RBIH Q4-21643?func=full-set-set\&set number $=008943 \&$ set entry $=000001 \&$ format $=999$

- Amador, C. (2018). Ventajas del liderazgo distribuido en instituciones de educación superior. Revista Iberoamericana para la Investigación y Desarrollo Educativo: RIDE, 8 (15), 817-832. Recuperado de: https://www.ride.org.mx/index.php/RIDE/article/view/322/1534

- Carrillo, M., Padilla, J., Rosero, T., \& Villagómez, M. (2009). La motivación y el aprendizaje. ALTERIDAD. Revista de Educación, 4 (2), 20-32. Recuperado de: https://www.redalyc.org/articulo.oa?id=467746249004

- Castellanos, S., \& Yaya, R. (2013). La reflexión docente y la construcción de conocimiento: una experiencia desde la práctica. Sinéctica, 41, 2-18. Recuperado de http://www.scielo.org.mx/scielo.php?script=sci arttext\&pid=S1665-109X2013000200006

- Cortes, A. (2005). Estilos de liderazgo y motivación laboral en el ambiente educativo. Revista Ciencias Sociales 6 (106), 2013-214. Recuperado de http://www.redalyc.org/html/153/15310615/

- Cermeño, A. (2016). La importancia de la motivación del profesorado en el aprendizaje del alumno. Trabajo de grado para optar el título de Lic. en Educación Primaria en Universidad de la Rioja, Logroño, La Rioja, España. Recuperado de: https://biblioteca.unirioja.es/tfe e/TFE001676.pdf

- Expósito, J. \& Manzano, B. (2010) Tareas educativas interactivas, motivación y estrategias de aprendizaje, en educación primaria, a partir de un curriculum modulado por nuevas tecnologías Teoría de la Educación. Educación y Cultura en la Sociedad de la Información, 11(1), febrero, 331-35. Recuperado de: http://www.redalyc.org/articulo.oa?id=201014897014

- Flores, E. (2010). El tipo de liderazgo del director y los estilos de comunicación de los docentes, estudiantes y personal administrativo del Instituto Superior Pedagógico Público "Nuestra Señora de Lourdes" DRE-Ayacucho. Tesis de grado para obtener Magister en Educación con Mención en Gestión de la Educación, Facultad de Educación, Universidad Nacional de San Marcos, Lima, Perú. Recuperado de: http://cybertesis.unmsm.edu.pe/handle/cybertesis/1675

- Goldrine, T., \& Rojas, S. (2007). Descripción de la práctica docente a través de la interactividad profesor-alumnos. Estudios pedagógicos (Valdivia), 33(2), 177197.Recuperado de https://scielo.conicyt.cl/scielo.php?script=sci arttext\&pid=S071807052007000200010

- González, O. \& González, L. (2012). Estilos de liderazgo del docente universitario. Multiciencias 12 (1), 35-44. Recuperado de: http://www.redalyc.org/pdf/904/90423275005.pdf

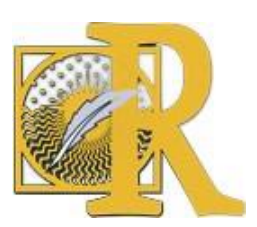


- Gutiérrez, J. (2008). ¿Cómo reconocemos a un buen maestro? Revista Mexicana de Investigación Educativa, 13 (39), 1299-1303. Recuperado de: http://www.redalyc.org/articulo.oa?id=14003914

- Ospina, J. (2006). La motivación, motor del aprendizaje. Revista Ciencias de la Salud, 4 (Esp), 158-160. Recuperado de: https://www.redalyc.org/articulo.oa?id=56209917

- Ospina Rave, B., Toro Ocampo, J., \& Aristizábal Botero, C. (2008). Rol del profesor en el proceso de enseñanza aprendizaje de la investigación en estudiantes de Enfermería de la Universidad de Antioquia, Colombia. Investigación y Educación en Enfermería, XXVI (1), 106-114. Recuperado de: http://www.redalyc.org/articulo.oa?id=105215231010

- Panta, R. (2010). Estilos de liderazgo predominante en docentes del nivel secundario de instituciones educativas públicas del distrito de Bellavista. Tesis para obtener el grado de Maestro en Educación con Mención de Evaluación y Acreditación de la Calidad de la Educación, Facultad de Educación, Universidad San Ignacio de Loyola, Lima, Perú. Recuperado de: http://repositorio.usil.edu.pe/handle/123456789/1265

- Ponce, R.A. (2008). El liderazgo y su relación con el rendimiento académico. Tesis para obtener el grado de Magister en Educación con Mención en Gestión Curricular, Facultad de Educación y Humanidades. Universidad del Bío-Bío, Chillán, Chile. Recuperado de: http://repobib.ubiobio.cl/jspui/bitstream/123456789/2125/1/Ponce Vidal Ricardo.pdf

- Prieto, L., \& Vera, L. (2008). Actitudes hacia la ciencia en estudiantes de secundaria. Psychologia: Avances en la Disciplina, 2 (1), 133-160. Recuperado de: http://www.redalyc.org/articulo.oa?id=297224999005

- Hernández, R., Fernández, C., \& Baptista, P. (2006). Metodología de la Investigación. México: Mc Graw-Hill Interamericana.

- Rivera, J., Cabazos, J. (2015). La importancia de la gestión y el liderazgo escolar en las instituciones de Educación Media Superior Tecnológica (CETIS y CBTIS) del estado de Tlaxcala. Revista Iberoamericana para la Investigación y el Desarrollo Educativo: RIDE, 6 (11), 359-380. Recuperado de: https://dialnet.unirioja.es/servlet/articulo?codigo=5280215

- Rojas, A., \& Gaspar, F. (2006). Bases de Liderazgo. Santiago, Chile: OREALC/UNESCO.

- Sierra, G. (2016). Liderazgo educativo en el siglo XXI, desde la perspectiva del emprendimiento sostenible. Revista Escuela de Administración de Negocios, (81), 111 -

128. Recuperado de; http://www.redalyc.org/articulo.oa?id=20649705007

- Silva, F., Gandoy., F., Jara, C., Pacenza, M.I. (2015). Liderazgo del docente y niveles de empoderamiento de los estudiantes en un seminario de prácticas comunitarias de una universidad pública argentina. Cuadernos de Administración, 31 (54), 68-79. Recuperado de: http://www.redalyc.org/pdf/2250/225044440008.pdf

- Steinmann, A., Bosch, B., \& Aiassa, D. (2013). Motivación y expectativas de los estudiantes por aprender ciencias en la universidad: Un estudio exploratorio. Revista Mexicana de Investigación Educativa, 18 (57), 585-598. Recuperado de: http://www.redalyc.org/articulo.oa?id=14025774012

- Tirado, F., Santos, G., \& Tejero-Díez, D. (2013). La motivación como estrategia educativa: Un estudio en la enseñanza de la botánica. Perfiles educativos, 35(139), 7992. Recuperado de: http://www.scielo.org.mx/scielo.php?script=sci arttext\&pid=S018526982013000100006\&lng=es\&tlng=es.

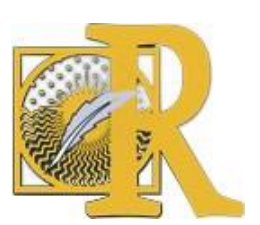

Fecha de recepción: 19-03-2019 Fecha de aceptación: 21-05-2019

Velázquez-Hernández, J. \& Hernández-Romero, G. (2020). Influencia del Liderazgo docente en la motivación de universitarios 
- Valenzuela, J., Muñoz, C., Silvia-Peña, L., Gómez, V., \& Precht, G. (2015). Motivación escolar: Claves para la formación motivacional de futuros docentes. Estudios pedagógicos (Valdivia), 41(1), 351-361. Recuperado de: https://scielo.conicyt.cl/scielo.php?script=sci arttext\&pid=S0718-07052015000100021

- Vilchez, Y. (2012). Ética y Moral: una mirada desde la gerencia pública. Revista Formación Gerencial, 11(2), 232-247. Recuperado de; http://produccioncientificaluz.org/index.php/rafg/article/view/656/658 


\begin{tabular}{|l|l|l|}
\hline Hola, te pido que por favor me apoyes a responder las \\
siguientes preguntas, las cuales servirán de apoyo para un \\
trabajo de investigación. \\
Ciclo/semestre:
\end{tabular}

1. El profesor explica de qué tratarán los temas que se abordarán en clases Muy de acuerdo De acuerdo Ni de acuerdo ni en desacuerdo Muy en desacuerdo

2. El maestro realiza preguntas para despertar el interés en las clases

Muy de acuerdo De acuerdo Ni de acuerdo ni en desacuerdo Muy en desacuerdo

3. El docente te motiva al logro de tus actividades

Muy de acuerdo De acuerdo Ni de acuerdo ni en desacuerdo Muy en desacuerdo

4. El profesor te inspira a que confíes en ti y en tus habilidades

Muy de acuerdo De acuerdo Ni de acuerdo ni en desacuerdo Muy en desacuerdo

5. El profesor usa ejemplos en los temas

Muy de acuerdo De acuerdo Ni de acuerdo ni en desacuerdo Muy en desacuerdo

6. El docente utiliza anécdotas o vivencias en sus explicaciones

Muy de acuerdo De acuerdo Ni de acuerdo ni en desacuerdo Muy en desacuerdo

7. El maestro muestra interés en tu progreso académico

Muy de acuerdo De acuerdo Ni de acuerdo ni en desacuerdo Muy en desacuerdo

8. El docente es tolerante y respetuoso ante una respuesta errónea

Muy de acuerdo De acuerdo Ni de acuerdo ni en desacuerdo Muy en desacuerdo

9. El profesor te motiva a ser mejor cada día

Muy de acuerdo De acuerdo Ni de acuerdo ni en desacuerdo Muy en desacuerdo

10. El profesor te ánima a tener éxito en tu vida

Muy de acuerdo De acuerdo Ni de acuerdo ni en desacuerdo Muy en desacuerdo

11. El docente tiene sentido del humor, haciendo sus clases interesantes

Muy de acuerdo De acuerdo Ni de acuerdo ni en desacuerdo Muy en desacuerdo 